\title{
La práctica de la interculturalidad en los estudiantes de cuarto año de Magisterio en Educación Intercultural Bilingüe ${ }^{[6]}$
}

Licda. Rosa Aura Palacios Rizo ${ }^{[7]}$

MA. Yuri Zapata Webb ${ }^{[8]}$

\section{Resumen}

Esta investigación valora la participación de la comunidad educativa en la práctica de la interculturalidad durante el proceso enseñanza - aprendizaje en los estudiantes de cuarto de Magisterio en Educación Intercultural Bilingüe de la Escuela Normal: "Gran Ducado de Luxemburgo", Bilwi, Puerto Cabezas, Región Autónoma del Atlántico Norte, en el año 2006.

El estudio es de tipo cualitativo y corte transversal, en él se aprecia la información referente a los roles desempeñados por los miembros de la comunidad educativa. Para lograr los objetivos planteados se realizaron entrevistas a madres y padres de familia, estudiantes, docentes, directora; además, se hicieron observaciones de clase directamente en el aula con el fin de registrar datos cualitativos durante el proceso educativo.

Entre los resultados obtenidos se destaca que la Escuela Normal cuenta con un currículo de formación para docentes en Educación Intercultural Bilingüe sustentado en las políticas educativas regionales: Autonomía, Identidad, Interculturalidad y Equidad de Género, lo cual presupone que la niñez debe apropiarse de su cosmovisión como base de las relaciones interculturales que se orientan hacia el conocimiento, respeto y valoración de las culturas de su pueblo, región y del país. Aunque la transformación del currículum fue un proceso participativo y de consenso entre actores del quehacer educativo para el desarrollo de la región; no obstante, se ha desistido al dejar todo en la iniciativa de docentes y estudiantes como únicos actores del proceso educativo en la escuela; mientras las madres y padres de familia, y otros líderes no hacen presencia en la misma.

\footnotetext{
[6] Monografía realizada con financiamiento de la fundación FORD con el proyecto Pathway II"Support to Graduation Research Projects of Indigenous and Afro Desendant Students in URACCAN".

[7] Licenciada en Ciencias de la Educación de la Universidad de las Regiones Autónomas de la Costa Caribe Nicaragüense -URACCAN, Recinto Bilwi.

[8] Director de Análisis y Planificación estratégica de la Universidad de las Regiones Autónomas de la Costa Caribe Nicaragüense. Tutor de la investigación.
} 
Asimismo, durante el proceso enseñanza - aprendizaje se ha hecho caso omiso a la práctica de la interculturalidad como eje transversal, a consecuencia que hacen falta estrategias que conlleven a la integración y prácticas sociales de la comunidad. La población que se atiende mayoritariamente es originaria de comunidades aledañas a Puerto Cabezas y Waspam, lo que indica que pertenecen a la etnia miskitu.

\section{Introduccion}

La educación en Nicaragua ha tenido cambios adheridos a las leyes, políticas y lineamientos del país. En la Costa Caribe nicaragüense en apego a la Ley de Autonomía y el Sistema Educativo Autonómico Regional (SEAR) se impulsó la transformación curricular para formación de docentes con enfoque Constructivista Humanista, que permita valorar su identidad étnica de manera que comprenda desde su cosmovisión: la idiosincrasia de los pueblos indígenas y comunidades étnicas, así como las relaciones culturales con otros pueblos de la región, el país y el mundo.

En la Costa Caribe nicaragüense, a partir del año 2003 se están aplicando los nuevos programas de estudio para la formación de Maestras y Maestros en Educación Intercultural Bilingüe -EIB-, por lo que el personal docente involucrado fue profesionalizado en dicha área con el fin de garantizar el desarrollo veraz y objetivo de las clases; no obstante, la práctica en el aula demostró que no es posible debido a la mínima participación de otras etnias. Esto debido a que el mayor porcentaje de población que se atiende es de la etnia miskitu, y en menor porcentaje mestiza, no teniendo presencia los demás pueblos indígenas y comunidades étnicas.

El modelo de Educación Intercultural Bilingüe en la Escuela Normal "Gran Ducado de Luxemburgo" ha tenido limitaciones para la implementación desde la bibliografía, como otros medios didácticos. Además, no se ha tenido la participación de los demás miembros de la comunidad educativa (madres, padres, líderes comunitarios, otros) en la práctica de la interculturalidad, por falta de planificación y comunicación entre la escuela con la comunidad para su involucramiento.

\section{Revision de literatura}

\subsection{Comunidad educativa}

Castillero (2002), indica que la comunidad educativa viene a ser el conjunto de actores sociales que tienen un interés particular en la educación, y esta puede estar expresada 


\section{EDUCACIÓN INTERCULTURAL BILINGÜE}

por el conjunto de actores sociales cuya interacción genera la consecuencia de estar vinculada a un centro específico y por una cierta cercanía geográfica.

Venecia (2002), afirma que la comunidad educativa está conformada por todas las personas, las familias e instituciones que contribuyen a la formación de las nuevas generaciones y al desarrollo educativo en un ámbito comunitario.

\subsection{Interculturalidad}

Heise (1999), afirma que la interculturalidad es una apuesta por el respeto a la pluralidad de racionalidades y a la heterogeneidad de formas de vida. La dimensión de la interculturalidad es particularmente necesaria en el campo de la educación para evitar choques traumáticos sobre la cosmovisión y los valores autóctonos impuestos por la cultura dominante. En tal sentido, el Sistema Educativo Autonómico Regional -SEAR- (2001) plantea que la interculturalidad promueve la valorización de las diversas culturas constituyendo herramientas pedagógicas científicas que contribuyan al diálogo, la articulación, la aceptación de otra u otro como legítimo, así las relaciones respetuosas entre los pueblos indígenas y comunidades étnicas de la Costa Caribe, entre ellos y el resto del país.

Kraine (1999), afirma que la interculturalidad es una dimensión que no se limita al campo de la educación, sino que se encuentra presente en las relaciones humanas en general, como alternativa frente al autoritarismo, dogmatismo y etnocentrismo, consecuentemente es un desafío vital, una actitud de apertura que libera de la tiranía de los prejuicios de las aparentes certezas absolutas.

Venezia (2003), refiere que la interculturalidad es la práctica que se establece entre diferentes personas, comunidades, pueblos y culturas que dialogan, intercambian saberes, crean juntos nuevos conocimientos en un marco de respeto y reconocimiento mutuo. Tiene su fundamento en el pluralismo, la voluntad, la experiencia y la convivencia en la diversidad. Para Rizo (2003) la interculturalidad es una propuesta educativa que promueve la revalorización de las diversas culturas, estableciendo herramientas pedagógicas científicas que contribuyan al diálogo, la articulación, la aceptación de la otra persona como legítima; promueve las relaciones respetuosas entre los pueblos de la Costa Caribe y el resto del país.

\subsection{La familia}

Para Gilspert (1999), la familia marca las pautas de conducta, valores y normas que permiten enfrentar al medio escolar donde se encuentra con una diversidad cultural, 
así como el medio laboral y otros espacios sociales. Los factores ambientales y sociales son las influencias más importantes en el modelamiento de la personalidad entre ellos el factor principal lo constituyen las relaciones interpersonales en la familia. La misma autora refiere que la madre y padre de familia deben formar actitudes y valores positivos, guiando a sus descendientes a que los practiquen en la vida diaria: alentándolos, estimulándolos y motivándolos, avivando el deseo e interés a la educación para enfrentar al medio escolar y otros espacios sociales donde la niña y el niño se sentirán en confianza y apoyados desde su hogar.

Kraine (1996), expresa que los padres de familia desempeñan un papel importante en la Educación Intercultural Bilingüe. Por cuanto ellos participan en su ejecución y colaboran con los docentes. Apoyan con sus conocimientos de la cultura, las técnicas y todas las tradiciones de la comunidad en el proceso educativo, a la vez, participan en las experiencias de la escuela, junto a sus niñas y niños.

\subsection{La escuela}

Según los programas de educación multicultural (2002), el papel de la escuela consiste en reconocer y respetar la diversidad étnica cultural, así como promover la cohesión social mediante el principio de participación de los grupos étnicos y culturales. De igual manera favorece la igualdad de oportunidades de todos los individuos, coadyuva a desarrollar y construir la sociedad sobre la base de la dignidad de todos de manera individual o colectiva, sobre la base del ideal democrático.

\subsection{Docentes}

Kraine (1996), afirma que para favorecer el proceso de interculturalidad el docente debe incorporar al aula los valores éticos y culturales de su propia comunidad y, a partir de cada individuo introducir el diálogo con otras culturas. Según Velásquez (12996), tenemos el caso de muchos maestros que en lugar de procurar conocer y entender la cultura de la población con la que trabaja, afronta una serie de conflictos al pretender imponer sus propios valores y conocimientos. Toda enseñanza de calidad requiere de docentes con claridad acerca de lo que va a enseñar, que se identifique con el quehacer pedagógico. No puede desechar experiencias y conceptos pedagógicos que podrían mostrar nuevos caminos de desempeño docente.

\subsection{Estudiantes}

La UCA-ASEN (2002), afirman que el alumno es el principal actor durante el proceso enseñanza - aprendizaje, esto permite avanzar en la medida que puedan construir 


\section{EDUCACIÓN INTERCULTURAL BILINGÜE}

significados adecuados en torno a los contenidos que configuran el currículo escolar, también incluye su aportación activa, su disponibilidad de conocimientos previos en el marco de una situación interactiva en la cual los docentes actúan como mediadores entre la niñez y su cultura, tal a como lo exige la diversidad de grupos étnicos en las circunstancias ante los que se encuentra.

\subsection{Identidad}

Kraine (2000), afirma que la identidad se manifiesta por rasgos sustanciales que hacen convertirse al individuo en una persona idéntica a sí, sueño de sí mismo y con capacidad autodeterminada. La identidad está siempre creándose, no es algo acabado ni puede llegar a serlo.

\subsection{Identidad cultural}

Ramírez (2002), refiere que la identidad cultural se configura desde el interior de la propia cultura, asimismo el proceso por el cual una persona se identifica, es decir, se siente parte de una cultura: respeta, valora, participa de sus manifestaciones, ceremonias, ritos, costumbres y tradiciones.

\subsection{Cultura}

Heise (1990), afirma que la cultura es un conjunto de formas y modos adquiridos de concebir el mundo, de pensar, hablar, expresarse, percibir, comportarse, organizarse socialmente, comunicarse, sentir y valorarse a uno mismo en cuanto individuo, en cuanto a grupo.

\section{Materiales y Métodos}

Se seleccionó el cuarto año de magisterio en Educación Intercultural Bilingüe de la Escuela Normal "Gran Ducado de Luxemburgo", debido a que fue uno de los primeros grupos con que se implementan los programas transformados en Educación Intercultural Bilingüe. Este estudio es de carácter cualitativo de corte transversal, con un universo de 38 estudiantes que representaron el total de $\mathrm{n}$ un mismo salón de clases. En esta misma sección fue donde había presencia de más de una etnia, mientras que en la otra sólo había estudiantes de la etnia miskitu. 
La muestra se seleccionó por conveniencia, que todos los involucrados de algún modo estuvieran relacionados a las acciones educativas, además que revelaran interés por contribuir con las prácticas interculturales en la Escuela Normal.

\subsection{Métodos y técnicas utilizadas}

Las fuentes primarias de investigación fueron: las entrevistas dirigidas a madres y padres de familia, estudiantes, docentes y directora de la Escuela Normal de esta región, así como observaciones directas a pie de aula durante el proceso enseñanza - aprendizaje, con el fin de valorar las estrategias utilizadas para contribuir en la práctica de la interculturalidad desde el aula de clase.

Previo a las entrevistas se facilitó el conocimiento en cuanto a los objetivos del estudio y las razones porqué se seleccionaron, destacando la importancia que tendría este estudio, ante lo cual accedieron muy amablemente.

Una vez obtenidos los datos requeridos, se procedió a la socialización y análisis de la información, afín de llegar a las conclusiones y plantear algunas recomendaciones.

\subsection{Aspecto ético}

Se aseguró a las personas informantes que los datos obtenidos no serían utilizados con fines de lucro, sino que el único propósito es contribuir con el desarrollo de la interculturalidad a través de la participación de la comunidad educativa en el proceso educativo. De igual manera se les aseguró preservar el anonimato de las personas que brindaron la información para este estudio.

\section{Resultados y discusión}

\subsection{Comunidad educativa}

En el estudio se maneja como comunidad educativa a todo el personal que de manera directa e indirecta deben estar involucrados en el proceso de enseñanza - aprendizaje, tales son: madres y padres de familia, estudiantes, docentes, líderes comunitarios, Ministerio de Educación. Sin embargo, en este estudio coinciden las personas entrevistadas en que se carece del personal de la comunidad que se haya involucrado en el proceso de formación magisterial, lo cual refleja que aún prevalece la concepción de que es el director y los docentes quienes deben controlar y conducir el proceso educativo. 


\section{EDUCACIÓN INTERCULTURAL BILINGÜE}

En la Escuela Normal "Gran Ducado de Luxemburgo", se carece de un inventario o registro (expedientes) sobre las fortalezas académicas, laborales y de oficios en relación a las madres y padres de familia, de igual manera que de las organizaciones, personalidades de la comunidad que podrían brindar apoyo para el desarrollo de los planes y programas de formación de docentes a través de conferencias, talleres, entre otros. Esto indica que los miembros de la comunidad educativa no están desempeñando su vitalidad creadora; careciéndose de su apoyo, tanto en conocimiento de cada una de las culturas, como del estímulo para que los estudiantes lleguen a construir ideas pedagógicas a través del aprendizaje colaborativo y cooperativo de manera consciente, a la vez que comparten y aprenden de manera recíproca.

También el papel central de la comunidad, las ancianas y ancianos, autoridades tradicionales y no tradicionales en todo el sistema de educación; no sólo es transmitir un saber tradicional, sino de participar en la elaboración de conocimientos nuevos a través del cual expresen la cosmovisión de los pueblos, lo cual se puede cumplir a través de conferencias, charlas, seminarios, talleres, diálogos, entre otros.

El estudio demuestra que la participación de la comunidad educativa es un elemento de mucha importancia en el nuevo modelo educativo para los pueblos indígenas y comunidades étnicas, puesto que deben participar de manera conjunta en el proceso educativo, así como en las gestiones, para que se pueda tener conciencia del tipo de educación que se ofrece a sus hijas e hijos; por tanto, todas las personas juegan un papel importante en el fortalecimiento de la interculturalidad.

\subsection{Interculturalidad}

Las maestras y maestros entrevistados expresaron que la práctica de la interculturalidad en el aula se ha efectuado mediante la creatividad para generar nuevas iniciativas como dramatizaciones, representaciones teatrales, cantos traducidos del español al miskitu. Cuando se celebran efemérides y actos culturales se presentan números culturales que representan las costumbres y creencias de los pueblos de la región. La interculturalidad es un proceso que involucra varios sistemas culturales que nace del derecho individual y colectivo de los pueblos indígenas, afrodescendientes, mestizos, así como la forma de cultivar las tradiciones, las expresiones culturales, valores y procesos educativos.

\subsection{Familia}

Las madres y padres de familia expresaron en su mayoría, que su participación en el proceso educativo es procurarles el tiempo necesario a sus hijas e hijos para que 
realicen sus tareas, mientras que en una menor escala refirieron que solamente brindan consejos para que estudien; no obstante, un trece por ciento manifestaron que les apoyan económicamente.

Por su parte la directora de la escuela expresó que existen disposiciones para la participación de las madres y padres de familia en los asuntos educativos; pero, que será en un futuro, está anuente a promover dicha integración a la vida educativa, ya que éstos sólo visitan la escuela cuando los citan por algún problema y algunas veces para retirar boletines informativos sobre las calificaciones obtenidas por el estudiante.

Lo anterior fue reafirmado por los docentes al referir, que solamente han visto madres o padres de familia cuando hay deficiencias por los estudiantes, menos que hayan recibido algún apoyo.

\subsection{Docentes}

Mediante las observaciones y entrevistas realizadas tanto a docentes como a la persona que dirige el centro de estudio, se revela que las maestras y maestros que atendieron el cuarto año de magisterio pertenecen a los grupos étnicos que tienen presencia en la Región Autónoma del Atlántico Norte; sin embargo, predominó el mestizo, cuya lengua materna es el español. Se destaca que tienen capacitaciones para desempeñarse en Educación Intercultural Bilingüe, específicamente con los nuevos programas para formación docente. No obstante, los acompañamientos reflejaron que durante el proceso enseñanza - aprendizaje, mientras las clases se desarrollaban en español, los estudiantes se comunicaban en su lengua materna, que es el miskitu.

Obviamente que el personal docente de Educación Intercultural Bilingüe en la Escuela Normal tienen adecuada formación académica y pertinente para su desempeño; antes bien, eso no es lo único para llenar las expectativas en esta modalidad por cuanto exige actitudes como respeto, tolerancia, tanto a sí mismo como a la sociedad a la que se debe. El ejemplo, no es solamente durante la actividad pedagógica en la escuela, sino ser sujeto activo y promotor de conciencia crítica, de modo tal que contribuya a la comunidad en el emprendimiento de ésta en la nueva modalidad educativa. Esto se confirma una vez que se hicieron observaciones y se constata que algunas clases son impartidas solamente en lengua miskitu, sabiendo que en el aula hay presencia de mestizos que no dominan otra lengua y en algunos casos es viceversa la situación.

Durante el proceso educativo de las clases de lenguaje y comunicación que comprende lengua materna y segunda lengua, la sección se divide por grupos étnicos con docentes para atender a cada etnia por su lengua, lo cual repercute negativamente 


\section{EDUCACIÓN INTERCULTURAL BILINGÜE}

porque se carece del intercambio de comunicación en las lenguas, a la vez, no se da la convivencia entre estudiantes durante el proceso de enseñanza - aprendizaje.

\subsection{Estudiantes}

El registro de matrículas que lleva la Escuela Normal se cotejó que durante el año lectivo 2006, se atendieron dos secciones de cuarto de magisterio en Educación Intercultural Bilingüe, entre las cuales, en la sección "A" solamente se atendieron estudiantes cuya etnia es miskitu; sin embargo, en la sección "B" fue donde se dio apertura a las otras etnias, se obtuvo una matrícula de 28 estudiantes que corresponden al $86 \%$ de la etnia miskitu y 5 mestizos con un 14\%. No obstante, al ser esta Escuela Normal formadora de maestras y maestros, la única para esta Región Autónoma, no se tuvo presencia de las demás etnias que tienen presencia en la región.

Lo anterior incide en la práctica de la interculturalidad, ya que las demás etnias carecen de presencia en la sección en estudio, por ende, para que se practique la interculturalidad deben darse relaciones entre las culturas, basadas en el respeto y desde los planes de igualdad, ya que esta no permite desigualdades entre culturas o que beneficien a un grupo cultural por encima de otros.

Si la práctica de la interculturalidad supone el reconocimiento de la multiculturalidad y de la multietnicidad de las Regiones Autónomas del país sustentada en el respeto, relaciones y valoración a la cultura, a la vez que, es la garantía de una convivencia armónica entre sujetos individuales o en colectivos de las distintas culturas, esto no se cumple en la formación de docente en Educación Intercultural Bilingüe en la Escuela Normal de esta región, debido a que no se han realizado acciones de captación de la población en edad estudiantil que tengan interés o vocación para ser docentes.

\section{Conclusiones}

En base a los resultados de esta investigación se presentan las siguientes conclusiones:

En la práctica de la interculturalidad durante el proceso educativo de la comunidad estudiantil del cuarto año de magisterio en Educación Intercultural Bilingüe, solamente tienen incidencias las personas estudiantes y docentes. El resto de los miembros de la comunidad educativa no han tenido participación. 
El currículo en Educación Intercultural Bilingüe para la formación de docentes de Educación Primaria tiene como uno de los ejes transversales la interculturalidad; empero, en la Escuela Normal "Gran Ducado de Luxemburgo", se ha hecho caso omiso al respecto, porque que se carecen de estrategias que conlleven a la integración y práctica social de la comunidad.

A pesar de tener un currículo definido para la formación de docentes en Educación Intercultural Bilingüe en la Costa Caribe nicaragüense, éste no es aplicado de manera efectiva porque se ha dejado únicamente en mano de docentes y estudiantes, mientras los demás miembros que integran la comunidad educativa, aún desconocen la responsabilidad que tienen con respecto a la formación de sus hijas e hijos.

La interculturalidad es uno de los ejes transversales en el currículo de formación de docentes en Educación Primaria Intercultural Bilingüe, éste no se practica a cabalidad debido a que la población que se atiende mayoritariamente es originaria de las comunidades aledañas a Puerto Cabezas y Waspam.

\section{Lista de referencias}

Compilaciones (2003). Medio ambiente, sociedad y desarrollo. Escuela Normal "Gran Ducado de Luxemburgo".

Castillero, Juan (2002). La Gestión en Educación. Cartago, Costa Rica.

Gilspert, Carlos. Enciclopedia General de Educación Tomo I y II. Editorial OCEANO, España.

Heise, María (1990). Interculturalidad un desafío. Edic. Lima Perú.

IPIL, URACCAN. -MED (2001). Sistema Educativo Autonómico Regional SEAR. $1^{\circ}$. Edición.

Kraine, Anita (1996). Educación Intercultural Bilingüe. $1^{\text {․ }}$. Edición.

Martine Abadía, Pretceille (1999). La Educación Intercultural. Colección Idea Books

Universitaria Educación. Barcelona. 


\section{EDUCACIÓN INTERCULTURAL BILINGÜE}

MECD (2002). Ley 413 Ley de Participación Educativa y su Reglamento. Gaceta No. 56 del 21 Marzo, 2002.

Ramírez Vargas, Margarita (2002). Tradición oral en el aula.

Rizo, Mario (2003). Educación Intercultural. 1º. Edición, Managua, Terra Nuova.

UCA ASEN. Marco Global y nacional de la Educación - Reforma Educativa. Managua, Nicaragua.

Velásquez de Bustamante, Martha Elizabeth. Pedagogía y Formación Docente. Primera edición. Carta Costa Rica.

Venezia Mauceri, Paolo (2003). Educación intercultural y Plurilingüe. $1^{\circ}$. Edición. Managua.Terra Nuova. 